\title{
L'exploitation Artisanale De L'or En Côte D'ivoire: La Persistance D'une Activite Illegale
}

\author{
Dr Goh Denis \\ Institut des Sciences Anthropologiques de Développement (ISAD) \\ Université de Cocody, Abidjan
}

doi: 10.19044/esj.2016.v12n3p18 URL:http://dx.doi.org/10.19044/esj.2016.v12n3p18

\begin{abstract}
The present article is the result of investigations to try to understand the persistence of illegal artisanal gold mining in Côte d'Ivoire where the phenomenon, although illegal, continues to grow for about twenty years. Relying on the case of the Yaouré area, the study was conducted mainly from the consultation of secondary data and groups and key informant interviews.

Survey data indicate that the illegal artisanal gold mining activity is a very lucrative business in which many players involved at various levels and with specific roles, are catered for at financially. The presence of people from the modern environment supposed to fight against clandestine gold mining continues certainly an explanatory factor for the persistence of this illegal activity itself.

Other causes for the development of illegal gold mining are the poor sales of traditional agricultural products (cocoa and coffee) and ignorance of the negative impacts of this activity on the biophysical and human environment by most of those involved in the sector.

It is clear from this study that ultimately illegal artisanal gold mining in the Yaouré, and certainly also in Ivory Coast, is a total social fact; Also, the fight against this phenomenon, to give the expected results, must not be limited to sporadic acts of repression against well diggers and other ore washers, weak link in the chain of illegal production of gold.
\end{abstract}

\section{Keywords:}

\section{Resume}

Le Présent article est le résultat d'investigations menées pour essayer de comprendre la persistance de l'exploitation artisanale clandestine de l'or en Côte d'Ivoire où le phénomène, bien qu'illégale, ne cesse de prendre de l'ampleur depuis environ vingt ans. S'appuyant sur le cas de la zone du 
Yaourè, l'étude a été menée essentiellement à partir de la consultation de données secondaires et des entretiens de groupes et d'informateurs clés.

Les données des enquêtes indiquent que l'activité d'exploitation artisanale clandestine de l'or est une activité très lucrative dans laquelle de nombreux acteurs, intervenant à des niveaux divers et avec des rôles précis, trouvent leur compte au plan financier. La présence des personnes issues du milieu moderne, censées lutter contre l'orpaillage clandestin, dans la filière d'exploitation artisanale de l'or, constitue certainement un facteur explicatif de la persistance de cette activité dite illégale.

Les autres causes du développement de l'exploitation clandestine de l'or sont la mévente des produits agricoles traditionnels (cacao et café) et l'ignorance des impacts négatifs à moyen et long termes de l'orpaillage sur le milieu biophysique et humain par la plupart des acteurs impliqués dans la filière.

Il ressort en définitive de cette étude que l'exploitation artisanale clandestine de l'or dans le Yaourè, et certainement ailleurs en Côte d'Ivoire, est un fait social total; aussi, la lutte contre ce phénomène, pour donner des résultats escomptés, ne peut-elle se limiter à des actions sporadiques de répression contre les puisatiers et autres laveuses du minerai, maillon faible de la chaine de production illicite de l'or.

\section{Mots clés :}

\section{Introduction}

L'exploitation artisanale de l'or, appelée aussi orpaillage, est une activité de type informel, exploitant cette ressource de manière non planifiée, en utilisant principalement des méthodes manuelles et recourant à des outils rudimentaires (approche de type "chasse-cueillette") (Jacques 2001).

Dans de nombreuses sociétés traditionnelles africaines, l'exploitation artisanale de l'or et donc la possession de ce métal précieux étaient motivées par les diverses fonctions socioculturelles que celui-ci jouait dans ces sociétés. L'or trouvé dans la nature ne devait pas être commercialisé car considéré comme un présent offert par les divinités et les génies et, il restait de ce fait, intimement lié à l'âme, à la fortune, à la destinée de celui qui l'a trouvé. (Niangoran-Bouah, 1978).

En Côte d'Ivoire, les premières traces de l'exploitation artisanale de l'or remonteraient au début de la deuxième moitié du $18^{\mathrm{e}}$ siècle; en effet, selon Joseph Gaston (1913), ce sont les travaux effectués par les indigènes de la Côte d'Ivoire pour exploiter l'or soit des filons, soit des gîtes alluvionnaires, qui ont été les premiers seuls guides de la prospection minière européenne dans cette colonie.

Aujourd'hui, les fonctions sociales que l'or jouait dans les sociétés traditionnelles ivoiriennes et qui justifiaient son acquisition semblent avoir 
disparu dans la quête de ce métal précieux; en effet, l'exploitation artisanale de l'or semble être motivée seulement par la recherche de revenu financier personnel, important et rapide. La "fièvre" de l'or a gagné tout le pays, attisant de plus en plus la convoitise de nombreux individus, de tous horizons. A ce propos, les données du Ministère de l'Industrie et des Mines (Actualité, 2014) indiquent qu'actuellement, la prolifération des sites d'orpaillage touche 24 régions sur les 31 que compte la Côte d'Ivoire et que cette activité est exercée par plus de 500000 personnes dans les milieux ruraux.

Si l'exploitation artisanale de l'or, au regard du fort engouement qu'elle suscite, peut paraître comme une activité lucrative, elle présente de nombreux impacts négatifs, tant au niveau social que biophysique. C'est pour maîtriser les effets néfastes de cette activité que l'Etat a soumis son exercice à autorisation. Cependant, force est de noter que de nombreux sites d'exploitation artisanale de l'or qui s'ouvrent et se développent en Côte d'Ivoire demeurent illégaux.

En dépit des discours officiels (qui annoncent des mesures vigoureuses de rationalisation des activités d'orpaillage) et de quelques "opérations coup de poing ${ }^{1 "}$ pour fermer des sites clandestins d'orpaillage, de nouveaux autres sites se créent et se développent régulièrement en dehors des conditions prévues par le code minier.

Pour ce qui est des facteurs explicatifs de la ruée des populations rurales vers l'or, deux raisons sont souvent évoquées: l'insuffisance des revenus agricoles (MARANDA D., 2011) et l'effondrement des cours des produits agricoles (KOUADIO K. N., 2008). Pour notre part, il semble opportun d'une part, de s'interroger sur la nature des acteurs en présence dans l'exploitation artisanale clandestine de l'or, ce qui pourrait expliquer la persistance d'un phénomène pourtant illégal, et d'autre part, d'essayer d'identifier les impacts de cette activité sur l'environnement.

C'est ce double objectif que le présent article vise à atteindre; il comprend trois parties principales:

- la présentation de la méthodologie de l'étude;

- l'identification des acteurs de l'exploitation artisanale clandestine de l'or;

- l'identification des impacts socioéconomiques et environnementaux de l'exploitation artisanale de l'or.

\footnotetext{
${ }^{1}$ Il s'agit de l'usage de la force publique pour fermer les sites d'exploitation artisanale clandestine de l'or
} 


\section{Methodologie}

\section{Le choix de la zone d'étude}

Le choix trois localités voisines d'Angovia, Allahou-Bazi et de Kouakougnanou, de la sous-préfecture de Bouaflé comme zone d'étude se justifie par quatre raisons:

- l'exploitation artisanale et clandestine de l'or constitue aujourd'hui la principale activité socioéconomique au niveau des trois localités citées et concerne pratiquement toutes les franges de la population (enfants, jeunes, vieux, autochtones, allochtones et allogènes, etc.);

- un impact négatif majeur de l'afflux de migrants, attirés par la fièvre l'or, a été durablement ressenti dans ces localités; en effet, en juillet 2013, un violent conflit a opposé les autochtones et les orpailleurs à Angovia, faisant officiellement 03 morts (les populations évoquent 20 cas de décès), de nombreux blessés et d'importants dégâts matériels (destruction d'une centaine de maisons, de nombreuses voitures et de motos, etc.);

- la mesure de fermeture des sites d'orpaillage ordonnée par les autorités ivoiriennes à la suite du violent conflit inter communautaire que la zone a connu en 2013 est restée sans effet; on a même assisté à l'ouverture de nouveaux sites d'exploitation artisanale de l'or dans ces trois localités;

- les activités d'orpaillage dans ces trois localités ont lieu sur un permis de recherche minière accordée par l'Etat de Côte d'Ivoire a un opérateur économique, ce qui est en violation des dispositions du décret $n^{\circ}$ 2014-397 du 25 juin 2014 déterminant les modalités d'application de la loi n 2014-138 du 24 mars 2014 portant Code Minier $^{2}$; à ce jour, rien ne semble être fait par les autorités pour mettre fin à cette situation qui dure depuis près de dix ans.

$\mathrm{Au}$ regard de ce qui précède, l'exploitation artisanale, dite clandestine, de l'or dans les localités d'Angovia, Allahou-Bazi et de Kouakougnanou met en lumière un paradoxe qu'il nous semble opportun d'élucider: le libre exercice et le développement d'une activité dite clandestine.

\section{La population enquêtée}

Le choix de la population enquêtée a été guidé par le souci d'avoir les opinions de tous les acteurs directement impliqués dans l'exploitation artisanale de l'or au niveau de 03 localités d'enquête. Ces acteurs sont:

\footnotetext{
${ }^{2}$ Selon l'article 64 de la Loi n 2014-138 du 24 mars 2014, les zones à l'intérieur desquelles l'exploitation minière artisanales est permises sont réservées ou déclassées dans des conditions déterminées par décret. Les activités d'orpaillage ne peuvent dont être menées sur d'un permis de recherche.
} 
- la notabilité des villages d'Angovia, Allahou-Bazi et de Kouakougnanou pour avoir accepté l'installation des orpailleurs dans leurs localités respectives: 03 groupes de 06 notables chacun, soit un total de 18 notables;

- les propriétaires terriens, principalement ceux qui ont autorisé l'exploitation de leurs parcelles à des fins d'orpaillage; 12 propriétaires terriens ont été interrogés dans le cadre de cette étude;

- 15 groupes d'orpailleurs, constitués de puisatiers et de laveuses du minerai: au total 93 orpailleurs ont fait l'objet d'enquête ;

- les financiers de la filière de l'exploitation artisanale de l'or: ce sont en quelque sorte les propriétaires des sites d'orpaillage. 02 personnes de cette catégorie d'acteurs se sont prêtées à nos questions.

$\mathrm{Au}$ total, 125 personnes ont constitué l'échantillon de personnes interrogées dans le cadre de la présente étude.

Au plan de la démarche, nous avons privilégié la méthode historique et la méthode systémique; la première nous semble appropriée pour connaître la genèse de l'exploitation artisanale de l'or dans la zone d'étude; la seconde méthode nous paraît indiquée pour non seulement identifier les facteurs explicatifs du phénomène étudié, mais aussi pour déterminer les interactions réciproques que les acteurs impliqués dans le phénomène de l'orpaillage entretiennent et qui rendent compte de la persistance d'une activité pourtant illégale.

Comme techniques de collecte des informations, nous avons eu recours à la recherche documentaire, aux entretiens et à l'observation.

Dans le cadre de la recherche documentaire, nous avons été confrontés à l'absence de données secondaires à caractère scientifique sur le sujet en Côte d'Ivoire. La recherche sur l'internet nous a permis d'avoir quelques données sur le sujet, notamment au Burkina Faso, en République Démocratique du Congo et en Guyane.

Deux types d'entretien ont été utilisés dans le cadre de la présente étude: les entretiens semi-directifs de groupe, avec pour cibles les notables, les puisatiers et les laveuses du minerai; les entretiens directifs individuels ont concerné, avec comme population cible les propriétaires terriens et les financiers de la filière d'exploitation artisanale et clandestine de l'or. Les entretiens semi-directifs de groupe et les entretiens individuels ont été menés à l'aide de guides d'entretien.

\section{Resultats de l'etude}

Les données d'observation et d'enquête permettent d'identifier les acteurs et leur rôle dans l'exploitation artisanale de l'or dans la zone d'étude, de déterminer les impacts de cette activité sur l'environnement, notamment sur le milieu humain. 


\section{Les acteurs et leur rôle dans l'exploitation artisanale de l'or}

L'exploitation artisanale de l'or dans les localités d'Angovia, AllahouBazi et de Kouakougnanou met en interaction une diversité d'acteurs dont les rôles, complémentaires, permettent à la filière de se maintenir. On peut distinguer globalement deux groupes d'acteurs: le groupe d'acteurs issus du milieu moderne et celui dont les membres proviennent du milieu rural.

\section{$\checkmark$ les acteurs de l'exploitation artisanale de l'or du milieu moderne}

De nos investigations, il ressort que les acteurs de l'exploitation artisanale de l'or du milieu moderne sont de deux types: les riches commerçants burkinabé, installés pour les uns à Abidjan, Daloa et à Yamoussoukro et, pour les autres, en dehors du territoire ivoirien, qui investissent financièrement dans les activités d'exploitation artisanale de l'or; ils ont tous la particularité d'être présents, depuis assez longtemps, dans cette activité. Quoique très peu connus de la plupart des orpailleurs opérant sur le terrain, ces acteurs sont les véritables "propriétaires" des sites d'orpaillage administrés pour leur compte, par des chefs de "chantier" présents sur le terrain.

Pour ce qui est des sites d'exploitation artisanale de l'or dans le Yaourè, nous avons identifié trois (03) principaux exploitants miniers d'origine burkinabé ne disposant d'aucune autorisation officielle d'exploitation artisanale de l'or, comme l'exige le code minier ivoirien; le seul document avec lequel ils travaillent et auquel nous avons eu accès est "une convention" d'exploitation de parcelle à des fins d'orpaillage qui les lie aux propriétaires terriens.

Le rôle de ces "hommes d'affaires", véritables propriétaires des sites d'orpaillage, est de créer les conditions d'ouverture et de fonctionnement d'un "chantier"; à cet effet, diverses charges leur incombent:

- le paiement des droits d'accès à la terre auprès des propriétaires terriens;

- le paiement des frais de divers sacrifices nécessaires pour que les génies rendent le "chantier" prospère et le préservent des accidents ou d'évènements malencontreux;

- la fourniture du matériel de travail aux orpailleurs;

- la prise en charge alimentaire et sanitaire des orpailleurs ${ }^{4}$;

- la prise en charge financière des "agents de sécurité ${ }^{5 "}$ lorsqu'il s'agit de grands "chantiers" comme « petit Abidjan ».

\footnotetext{
${ }^{3}$ Les populations locales et les orpailleurs appellent "chantier", les sites d'orpaillage

${ }^{4}$ La durée de la prise en charge sanitaire des orpailleurs est celle de la vie du "chantier"; la prise en charge alimentaire couvre la période où le "chantier" n'est pas encore productif.

${ }^{5}$ il s'agit d'individus (généralement des chasseurs traditionnels appelés dozos) recrutés et armés de fusils et d'armes blanches pour, dit-on, assurer la sécurité sur le "chantier". Sur le "chantier" de petit Abidjan à Kouakougnanou, il y aurait, selon les données fournies par le
} 
C'est à propos de l'importance du rôle des propriétaires des "chantiers" qu'un notable de Angovia a déclaré ceci: "N'ouvre pas un chantier qui veut, mais qui peut: le travail de l'or demande beaucoup de moyens et il faut avoir beaucoup d'argent pour le faire".

Outre les riches commerçants burkinabé, on a, comme acteurs de l'exploitation artisanale de l'or du milieu moderne, les personnes dites de pouvoir, ou d'autorité: ce sont des agents et/ou des responsables des forces de défense sociale, des responsables de l'administration publique locale. Bien que ces acteurs n'investissent pas financièrement dans l'orpaillage, ils constituent un maillon important de la filière; en effet, c'est avec leur caution tacite et leur bienveillance, que l'exploitation artisanale clandestine de l'or se met en place et se maintient.

Comme l'on peut le deviner, l'intervention des personnes dites de pouvoir dans l'exploitation artisanale de l'or reste cachée et vise au moins deux objectifs complémentaires: accorder une apparence de légalité à une activité clandestine et, par ricochet, permettre aux propriétaires des "chantiers" d'opérer sans trop de problèmes avec les populations rurales.

Les accointances des responsables administratifs avec le milieu de l'orpaillage clandestin semblent évidentes quand on considère l'importance, en nombre et en taille, des sites d'exploitation clandestine de l'or dans les départements de Bouaflé et de Yamoussoukro. Nombre de ces sites sont contigus aux grandes voies de communication et, jusqu'à ce jour, les orpailleurs opèrent dans un climat de paix continue.

En dehors des acteurs du milieu moderne, l'exploitation artisanale clandestine de l'or dans le Yaourè mobilise de nombreux autres acteurs de terrain ayant chacun un rôle précis.

\section{$\checkmark$ les acteurs de l'exploitation artisanale de l'or du milieu rural}

Les acteurs intervenant directement, sur le terrain, dans l'exploitation artisanale clandestine de l'or sont les chefs des "chantiers", les propriétaires terriens et les orpailleurs.

Sur chaque site d'orpaillage, le chef du "chantier" représente le propriétaire du site pour le compte de qui il organise la chaîne de production de l'or: le recrutement et l'entretien des orpailleurs, l'achat du matériel d'orpaillage, le contrôle de la production de l'or, la supervision de l'achat de l'or produit, la gestion de la sécurité sur le site d'orpaillage, etc. C'est le chef du "chantier" qui, du fait de la non présence du propriétaire du "chantier" sur le site, a la responsabilité de créer les conditions d'une exploitation viable du site.

responsable dudit "chantier", 36 "agents de sécurité payés mensuellement par le propriétaire du "chantier".. 
Les propriétaires terriens constituent des acteurs importants dans la filière d'exploitation artisanale clandestine de l'or; il convient de noter que la qualité de propriétaire terrien relève du droit coutumier local suivant lequel les terres appartiennent à des villages, mais de façon plus précise, à des lignages et à des familles à l'intérieur des villages. C'est donc le chef de lignage ou de famille qui fait office de chef de terre qui, reste un bien commun inaliénable.

Le rôle des chefs de terre dans l'exploitation artisanale de l'or consiste à autoriser, suivant des modalités négociées avec le futur propriétaire de "chantier" ou le représentant de celui-ci, l'accès à leur patrimoine foncier à des fins d'orpaillage. C'est également à eux qu'il revient le devoir de faire les sacrifices et autres libations dédiés aux génies et aux mânes des ancêtres avant l'ouvertures des "chantiers" et aussi durant son fonctionnement quand cela s'avère nécessaire ${ }^{6}$.

Les orpailleurs constituent la frange d'acteurs la plus importante, au plan numérique, intervenant dans l'exploitation artisanale clandestine de l'or. Dans le cas de l'exploitation d'un filon d'or, l'équipe d'orpailleurs est constituée soit uniquement de creuseurs, soit de creuseurs et de concasseurs. Dans le premier cas, le concassage des pierres se fait à l'aide de machines installées sur ou hors du site d'orpaillage et dans le second, le broyage des pierres se fait manuellement ou à l'aide de machines sur le "chantier" (photos ci-dessous).

Pour ce qui est de l'exploitation artisanale de gîtes alluvionnaires, elle mobilise une équipe composée d'une dizaine de personnes, travaillant à la chaîne, en deux groupes différents. Le premier groupe est constitué de trois à quatre personnes; ce sont des puisatiers, chargés de creuser la mine à tour de rôle et à faire remonter le minerai du puits à l'aide d'une poulie ou par simple traction humaine. C'est la tâche la plus harassante de l'activité d'exploitation artisanale de l'or, avec des risques d'éboulement de terrain. Elle est exécutée uniquement par des hommes.

La deuxième composante des orpailleurs est constituée par des femmes (02 à 03 personnes) chargées de laver le minerai fourni par les puisatiers (photo $\mathrm{n}^{\circ} 3$ ). En général, les orpailleurs travaillent sous la surveillance étroite des représentants du propriétaire terrien et du chef du "chantier" dont le rôle est d'empêcher le vol de l'or produit.

\footnotetext{
${ }^{6}$ Diverses situations rendent nécessaires des sacrifices durant la vie d'un "chantier": ce sont notamment la baisse de la production de l'or, les bagarres et rixes avec effusion de sang, les conflits entraînant l'intervention des personnes étrangères au village et à la zone du "chantier", etc.
} 


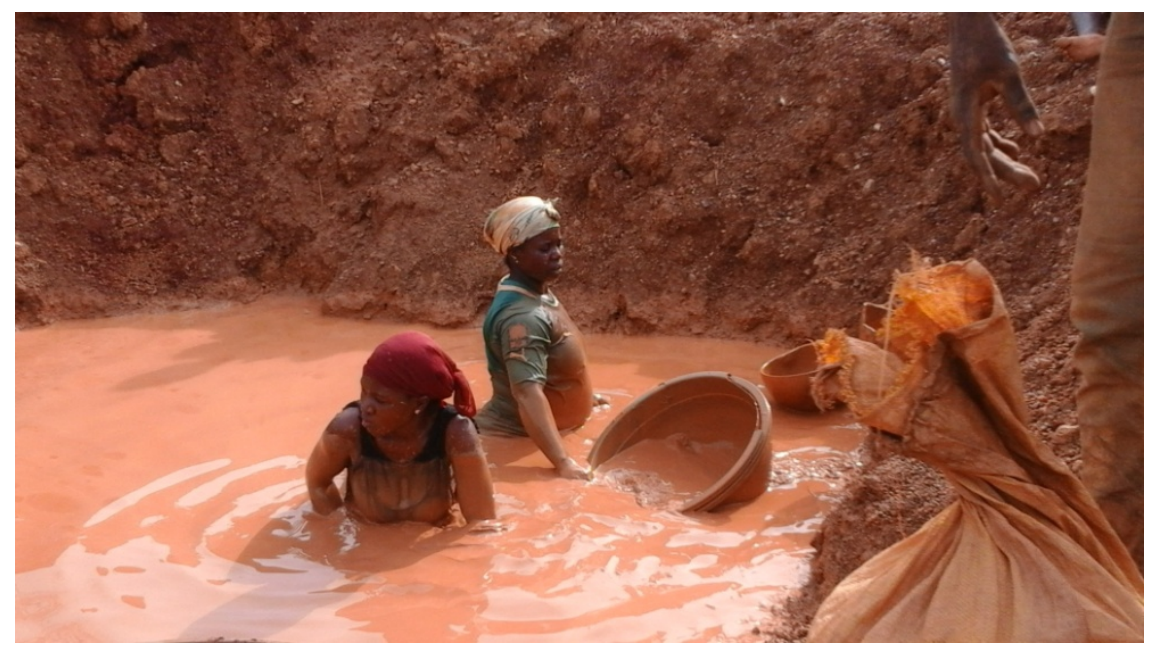

Photo 3: les laveuses du minerai

L'exploitation artisanale clandestine de l'or dans le Yaourè présente aussi bien des impacts positifs que négatifs sur les composantes de l'environnement. Il convient de préciser que cette étude ne s'intéresse qu'aux impacts sociaux de cette activité; toutefois, l'utilisation des produits comme le mercure par les orpailleurs laisse supposer que l'exploitation artisanale clandestine de l'or a des effets négatifs non seulement sur les milieux récepteurs, mais également sur la santé des populations.

\section{Les impacts positifs de l'exploitation artisanale clandestine de l'or}

Les impacts positifs de l'exploitation artisanale clandestine de l'or sur le milieu social concernent les gains financiers que les propriétaires terriens ont, en contrepartie de la cession de leur patrimoine foncier à des fins d'orpaillage, les emplois générés par la filière et le développement du petit commerce dans la zone d'exploitation artisanale clandestine de l'or.

\section{$\checkmark \quad$ les gains financiers perçus par les propriétaires terriens}

Les gains financiers perçus par les propriétaires terriens sont de deux types: les droits d'accès à la terre et la quote-part perçue de la vente de l'or produit sur leur parcelle.

Les droits d'accès à la terre sont des sommes d'argent, payées d'un trait, aux propriétaires terriens par quiconque souhaite ouvrir un site d'orpaillage. Le droit d'accès à la terre, qui n'est pas le prix de vente de la parcelle à exploiter, est d'un montant variable et se négocie en fonction de la superficie du terrain, de la qualité supposée ou réelle de celui-ci (importance des indices de présence de l'or) et de la demande (nombre de personnes intéressées par le site en question).

Le droit d'accès à la terre dans la zone a beaucoup évolué dit-on, et est aujourd'hui compris entre 300000 et plusieurs millions de francs CFA; 
ainsi par exemple, selon les informations fournies par le chef du "chantier" de «petit Abidjan », ce site d'orpaillage a été obtenu contre payement de la somme de 3000000 francs CFA! Cette information, confirmée par le propriétaire du terrain, montre l'importance des gains financiers que la cession d'un lopin de terre à des fins d'orpaillage peut procurer à son propriétaire.

Outre le droit d'accès à la terre, les propriétaires terriens perçoivent une partie des revenus tirés de la vente de l'or produit sur leur parcelle. Les gains perçus ici sont fonction de la quantité d'or exploité; ainsi, pour chaque gramme d'or produit, le propriétaire terrien reçoit de l'exploitant de la parcelle, une somme de 2000 francs CFA. Comme on peut le constater, les revenus du propriétaire terrien sont liés à la qualité de sa parcelle cédée à des fins d'orpaillage; aussi, n'hésite-t-il pas, quand cela est nécessaire, (généralement à la demande du chef du "chantier") de procéder à des sacrifices ou à des libations pour demander aux génies gardiens de l'or, de libérer le précieux métal sur sa parcelle.

$\checkmark$ Les emplois générés par l'exploitation artisanale clandestine de l'or

L'exploitation artisanale clandestine de l'or génère des emplois directs et des emplois indirects; les premiers concernent le creusage des puits, le lavage du minerai, le concassage manuel des pierres, la gestion du "chantier" et la gestion de comptoirs d'achat d'or. Si les gains tirés de l'exploitation artisanale clandestine de l'or paraissent aléatoires et difficilement mesurables, il est indéniable que cette activité occupe à plein temps de nombreux individus dont certains semblent en vivre.

Le nombre de personnes intervenant dans les activités d'orpaillage, en qualité d'employés n'a cessé d'augmenter depuis l'ouverture des premiers "chantiers" dans le Yaourè au point où l'exploitation artisanale clandestine de l'or y est devenue, de l'avis de toutes les personnes interrogées, la première activité socioéconomique. A ce propos, le chef du village de Kouakougnanou a déclaré ce qui suit: "Grâce au travail de l'or, nos enfants se débrouillent très bien; c'est grâce à ça qu'ils arrivent à s'occuper de nous et à construire toutes ces belles maisons que vous voyez dans ce village."

Comme le témoignent les propos ci-dessus, l'exploitation artisanale clandestine de l'or occupent de nombreux jeunes et constituent pour eux aujourd'hui la principale, voire l'unique source de revenu.

\section{$\checkmark$ le développement des activités commerciales dans la zone d'orpaillage}

Le développement de l'exploitation artisanale clandestine de l'or dans le Yaourè est allé de pair avec celui de nombreux besoins dont la recherche de satisfaction a eu pour effet premier le développement des activités commerciales dans les villages et sur les sites d'orpaillage; ces besoins sont essentiellement ceux de nourriture et de matériels de travail. 
Le commerce des denrées alimentaires, notamment la restauration populaire, semble être florissant dans les villages du Yaourè et sur les sites d'orpaillage; en effet, la présence de nombreux individus attirés par la fièvre de l'or et se déplaçant généralement seuls a pour corollaire le développement des besoins en nourriture; les nombreuses gargotes présentes sur les sites d'orpaillage et dans les villages essayent de satisfaire cette forte demande de nourriture.

L'exploitation artisanale clandestine de l'or a aussi permis l'ouverture de fabriques, de boutiques et d'ateliers de réparation d'outils et matériels de travail des orpailleurs (bèches, pelles, pics, pioches, marteaux, pièces métalliques diverses, bouteilles de gaz sciées, motopompes, torches, lampes tempête, poulie, cordes, concasseurs) qui constituent à ce jour les sources de revenu de nombreuses chefs de familles.

Les informations ci-dessus montrent que l'exploitation artisanale clandestine de l'or, du fait du développement du commerce et de l'artisanat qu'elle engendre, donne une certaine vitalité à l'économie locale. Les impacts positifs de l'exploitation artisanale clandestine de l'or ne peuvent toutefois occulter les effets négatifs de cette activité.

\section{Les impacts négatifs de l'exploitation artisanale clandestine de l'or}

Les impacts négatifs de l'exploitation artisanale de l'or au niveau du Yaourè sont nombreux et observables sur les équilibres sociaux et communautaires, l'économie locale, la santé et la sécurité des acteurs au travail, l'éducation et sur les groupes vulnérables, notamment les femmes et les enfants.

\section{$\checkmark$ la fragilisation des équilibres sociaux et communautaires}

Les données de l'Etude d'Impact Environnemental et Social (EIES) commanditée par Cluff Gold en 2006 faisaient état de la présence de 600 orpailleurs à Alahou-Bazi et à Angovia. Les observations faites dans le cadre de la présente étude tendent à montrer que ces données sont largement dépassées: les localités de Allahou-Bazi et d'Angovia semblent avoir vu leur population doubler; le site de Petit Abidjan comptait, deux mois après son ouverture en 2014, plus de 2000 personnes $^{7}$.

Cette importante concentration de populations allogènes, dans un milieu où elles manquent d'ancrage socioculturelle, entraine la perturbation de l'équilibre social local avec de régulières tensions intercommunautaires dont l'une, particulièrement violente, en juillet 2013, a occasionné de nombreux dégâts matériels et des pertes en vies humaines.

Une autre conséquence négative de la forte concentration de populations consécutive à l'ouverture des sites d'orpaillage est l'apparition de

\footnotetext{
${ }^{7}$ Il s'agit d'une estimation faite par le chef du "chantier" petit Abidjan.
} 
comportements déviants, notamment la prostitution et la consommation excessive d'alcool. Concernant ce dernier élément, l'on note une prolifération de buvettes, bars et autres points de vente d'alcool de toutes les natures.

L'on observe également que l'augmentation rapide de la population accroit la pression humaine sur les infrastructures sociales de base en place; ainsi par exemple, à Angovia et à Allahou-Bazi (les deux localités sont contigües), principales zones d'installations des orpailleurs, les points d'eau potable, le groupe scolaire et le centre de santé répondent aujourd'hui difficilement aux sollicitations des populations dont le nombre a plus que triplé en moins de dix (10) ans ${ }^{8}$.

L'exploitation artisanale clandestine de l'or génère, chez certains acteurs locaux, des revenus financiers importants qui favorisent le renversement partiel des hiérarchies sociales établies depuis toujours dans les villages; en effet, les jeunes autochtones ayant fait fortune grâce à cette activité sont plus l'objet de considération sociale que les anciens; on note également que de riches chefs de "chantiers" allogènes, du fait de leur pouvoir économique, deviennent des contrepoids au pouvoir des autorités traditionnelles autochtones.

Un autre impact négatif de l'exploitation artisanale clandestine de l'or perceptible dans la zone d'étude est la fragilisation de nombreuses cellules familiales: le pouvoir économique des orpailleurs (majoritairement allogènes et se déplaçant en général sans leurs conjointes) constitue pour eux une arme de séduction et donc un facteur de perturbation des équilibres intra familiaux; cet impact négatif affecte de la même manière les rapports entre des communautés.

\section{$\checkmark$ le développement des problèmes de santé et de sécurité liés au travail des orpailleurs}

Il n'existe, à notre connaissance, aucune donnée d'étude relative à l'impact sanitaire des activités d'orpaillage. Toutefois, les observations de terrain montrent que cette activité est de nature à affecter de façon négative la vie et la santé des orpailleurs et celle des populations riveraines pour plusieurs raisons; en effet, l'exploitation artisanale de l'or, telle qu'elle se fait dans le Yaourè semble à la fois dangereuse et pénible pour plusieurs raisons:

- les accidents de travail mortels, semble-t-il nombreux, mais cachés pour éviter que les autorités administratives ne ferment les "chantiers" et aussi à cause des croyances populaires suivant lesquelles les éboulements sur les sites d'orpaillage, lorsqu'ils sont

\footnotetext{
${ }^{8}$ les données des enquêtes socioéconomiques que nous avons réalisées en 2008 et 2015 dans le cadre des études d'impact environnemental et social du projet minier de Cluff Gold et de Amara-Mining et l'analyse des données du Recensement Général de la Population et de l'Habitat de 1998 et de celui de 2015 semblent indiquer que la population de ces deux localités a été multipliée par 4 en moins de 10 ans.
} 
meurtriers, attirent les filons d'or et rendent ainsi le "chantier" rentable;

- l'absence de règles élémentaires de sécurité et d'hygiène sur les sites d'orpaillage: l'environnement des sites d'orpaillage se caractérise par la précarité des conditions de travail (absence de matériel de protection individuel) et l'utilisation de produits hautement toxiques, tels que le mercure;

- le développement de la prostitution avec pour corollaires les infections sexuellement transmissibles (IST) dans la zone d'étude. Concernant les IST, les données de l'enquête socioéconomiques réalisée en $2007^{9}$, indiquait déjà une prévalence du VIH-SIDA assez élevée chez les femmes enceinte à Angovia et à Allahou-Bazi.

Il apparaît également que les personnes intervenant dans l'exploitation artisanale de l'or sont exposées aux affections pulmonaires liées aux poussières, aux risques de surdité à cause des bruits permanents de machines et des marteaux utilisés pour le concassage et le broyage des pierres, aux risques de blessures par les éclats de pierre dans les yeux et aux affections oculaires.

\section{$\checkmark$ la précarisation de l'économie locale et des conditions de vie des orpailleurs}

Les revenus générés par l'exploitation artisanale clandestine de l'or sont appelés "l'argent rapide" par les populations, en comparaison aux moyens financiers que leur procurent les activités agricoles traditionnelles (culture de cacao et de café notamment). A ce propos, un propriétaire terrien interrogé à Allahou-Bazi a déclaré ceci: "Faire une plantation de cacao prend beaucoup de temps et demande du travail! Or, si tu donnes ta forêt aux "clandos"10 , rapidement tu gagnes beaucoup d'argent".

Cet avis rapporté ci-dessus est partagé par onze des douze propriétaires terriens interrogées; seul un propriétaire terrien, dont une parcelle est pourtant exploitée à des fins d'orpaillage, a semblé d'avis contraire. Aujourd'hui, l'on peut affirmer que l'exploitation artisanale clandestine de l'or est la principale activité économique dans les localités de Angovia, Allahou-Bazi et de Kouakougnanou. Cette situation a des effets négatifs sur l'économie locale.

La première conséquence négative, au plan économique, de l'abandon de l'agriculture au profit de l'exploitation artisanale de l'or est l'inflation des prix des denrées alimentaires; les populations de base reconnaissent cette

\footnotetext{
${ }^{9}$ Données de l'Etude d'Impact Environnemental et Social du projet minier d'Angovia, 2007.

${ }^{10}$ Les populations appellent "clandos" les orpailleurs, certainement en référence au caractère clandestin de leurs activités
} 
situation et affirment unanimement que "l'or a gâté le marché ici; tout est devenu cher".

L'on note également la précarisation des revenus des populations rurales; en effet, bien que de nombreux paysans tirent directement des revenus de l'exploitation artisanale clandestine de l'or, il est indéniable que ces revenus ne sont ni fixes, ni durables. Cette situation amène les propriétaires terriens à céder leurs parcelles, les unes après les autres, aux orpailleurs après l'épuisement des gisements des premières.

Une autre conséquence de l'exploitation artisanale clandestine de l'or, liée à l'extension et ou à la multiplication des sites d'orpaillage est l'amenuisement des terres et des superficies des exploitations agricoles; en effet, en exerçant cette activité aussi bien dans les jachères que dans les plantations en exploitation, les orpailleurs réduisent les disponibilités de terres cultivables et la viabilité des exploitations agricoles. Cette situation est d'autant plus préoccupante que les sites d'exploitation artisanale clandestine de l'or ne font pas l'objet de réhabilitation.

L'impact négatif de l'exploitation artisanale clandestine de l'or s'observe également au niveau du cadre de vie orpailleurs et des populations hôtes: au niveau d'Allahou-Bazi et d'Angovia, l'afflux des orpailleurs a entrainé la prolifération des "Mananbougou", habitat précaire, constitué de bois couverts avec des bâches-plastique, sans latrines (photo 4). L'importance de cet habitat précaire donne aux deux villages, l'image de localités engorgées et exposées aux risques liés à une mauvaise gestion des déchets ménagers et au péril fécal.

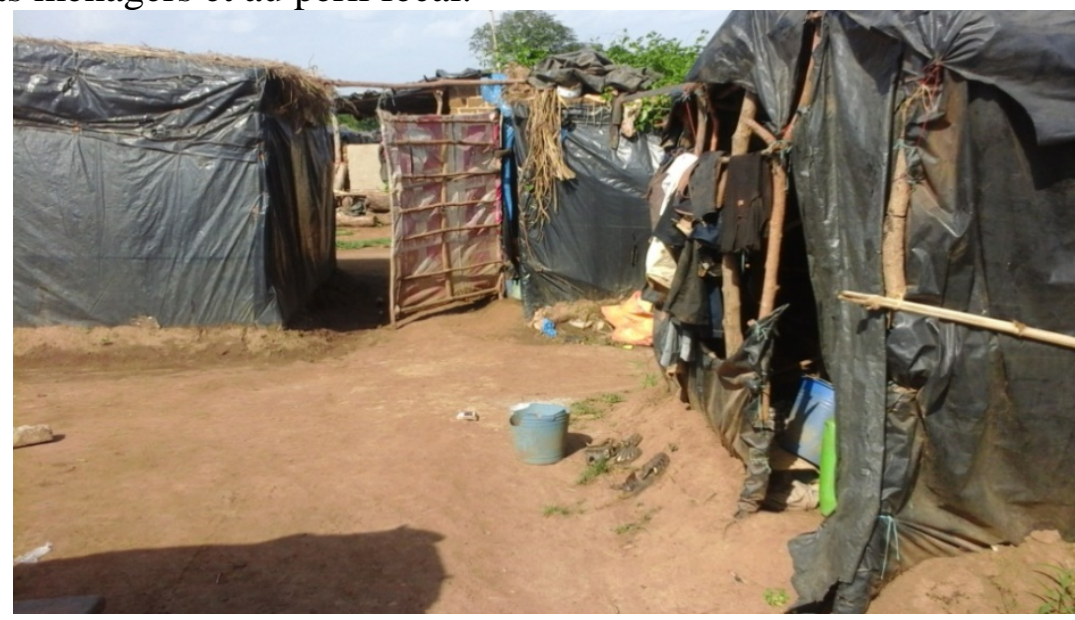

Photo 4: le "Manabougou" ou maison des orpailleurs

\section{$\checkmark \quad$ l'augmentation du niveau de déperdition scolaire}

Compte tenu du fait que l'exploitation artisanale clandestine de l'or est considérée par les populations du Yaourè comme "l'argent rapide", de 
nombreux jeunes gens préfèrent tenter leur chance sur les sites d'orpaillage plutôt que de poursuivre des études dont les perspectives d'emploi et de revenu sont, à leurs yeux, incertaines. C'est dans ce sens qu'un jeune homme rencontré sur un "chantier" à Angovia a déclaré ceci: "J'ai abandonné l'école en classe de terminale pour venir me débrouiller ici et m'occuper de mes parents; je ne me plains pas de ce que je gagne ici; l'important, c'est que je gagne ma vie."

Quand des familles ne poussent pas leurs enfants à abandonner les cours au profit des "chantiers", elles les encouragent à aller se débrouiller sur les sites d'orpaillage les jours fériés et pendant les vacances scolaires. Cette situation a des conséquences négatives sur le rendement des jeunes apprenants. Au regard de ce qui précède, il apparaît que la présence de nombreux sites d'exploitation artisanale clandestine de l'or dans le Yaourè y accroit le niveau de déperdition scolaire.

\section{$\checkmark \quad$ l'aggravation des conditions de vie des groupes vulnérables}

Les groupes vulnérables que constituent les enfants et les femmes sont très présents dans la chaîne de production de l'or.

Concernant les enfants, ils interviennent dans la filière d'exploitation clandestine de l'or soit pour leur propre compte, soit pour le compte de leurs parents. Dans le premier cas, les enfants offrent leurs services aux gestionnaires des "chantiers" et leur travail consiste à transporter le minerai du puits aux sites de lavage ou à concasser et à tamiser le minerai. La rémunération journalière des enfants est variable, mais atteint rarement 2000 francs CFA.

Les enfants qui travaillent pour le compte de leurs parents représentent les propriétaires terriens; ils sont chargés de surveiller la production de l'or, notamment à l'étape du lavage du minerai, pour empêcher le vol du métal précieux. La présence des enfants ici semble nécessaire dans la mesure où le revenu du propriétaire est fonction de la quantité d'or produite sur la parcelle qu'il a cédée.

En dehors des deux raisons ci-dessus évoquées, on retrouve sur les sites d'orpaillage des enfants qui accompagnent leurs parents; il s'agit des enfants des femmes employées pour le lavage du minerai et pour le concassage des pierres. La présence des enfants sur les sites d'exploitation artisanale clandestine de l'or les expose à des risques d'intoxication par des produits utilisés par les orpailleurs pour libérer le précieux métal; ainsi par exemple, l'intoxication au mercure est de nature à causer des handicaps permanents pour des enfants.

Pour les enfants intervenant directement dans l'exploitation de l'or, le travail dans ces conditions difficiles mettent en danger leur santé, leur croissance, leur sécurité, leur développement et leur dignité ou leur moralité; 
aussi, est-on en droit d'affirmer que le travail des enfants dans l'exploitation artisanale de l'or constitue pour ceux-ci des pires formes de travail.

S'agissant des femmes (de même que les jeunes filles), il convient de préciser qu'au niveau du Yaourè, elles ne descendent pas dans les puits. En dehors de quelques rares d'entre elles qui sont propriétaires de "chantier" de petite taille qu'elles gèrent au quotidien, le travail des femmes dans l'exploitation artisanale clandestine de l'or ici se compose du transport et du lavage du minerai, du concassage et du tamisage des pierres.

En dehors du travail direct dans la chaine de production de l'or, on retrouve, sur les sites d'orpaillage, les femmes dans le petit commerce: vente de nourriture, de boissons alcoolisées, de cigarettes et de divers menus articles. Toutefois, des témoignages concordants indiquent qu'en dehors de ces activités officielles, la prostitution est devenue une source de revenu pour de nombreuses filles, parfois âgées d'à peine 12 ans.

La présence des femmes sur les sites d'exploitation artisanale clandestine de l'or favorise le développement de la prostitution d'au moins deux manières: la première est la situation des femmes (ou jeunes filles) venues chercher du travail sur les sites d'orpaillage et qui n'en trouvent pas ou, quand elles en trouvent, en tirent un revenu insuffisant pour vivre décemment; ces femmes, n'ayant pas d'autres alternatives, s'orientent vers la prostitution.

Le second moyen par lequel les femmes arrivent à la prostitution est le biais des propriétaires des bars et autres "maquis" assez nombreux à Angovia et à Allahou-Bazi: les jeunes recrutés pour travailler dans ces lieux finissent par s'adonner au commerce de leur corps, soit par contrainte, soit par option.

Il convient de noter que deux éléments en rapport étroit avec à l'exploitation artisanale clandestine de l'or rendent la demande sexuelle particulièrement forte: la mobilité des orpailleurs qui se déplacent sans leurs conjointes et des pratiques magico-religieuses associées à la recherche de l'or.

Concernant ce dernier point, il faut comprendre que dans le contexte général des sociétés traditionnelles africaines, l'or n'est une créature simple, mais le métal des métaux, le plus notable des métaux et possédant un esprit fort et redoutable. Etre vivant, l'or ne demeure pas en place dans la nature, il se déplace d'un point à un autre de la terre. Il peut se rendre visible et invisible. (Niangoran-Bouah, 1978). Aussi, toute quête de l'or, pour être fructueuse, demande-t-elle l'observation de rites dont certains ont un caractère magico-religieux.

Le rite magico-religieux, ayant un rapport avec la prostitution, qui semble le plus usité, si l'on s'en tient à des témoignages recueillis auprès de propriétaires terriens et confirmés indirectement par des orpailleurs est le fait 
de ces derniers; il consiste pour eux (surtout les creuseurs), à avoir des rapports sexuels avec une partenaire, après une journée de travail à la mine et sans s'être lavé auparavant, à rester tel toute la nuit et à aller travailler le lendemain à la mine!

Cette pratique difficilement concevable, au regard des problèmes d'hygiène corporelle et de santé qu'elle pose, serait acceptée par de nombreuses jeunes femmes; en effet, le payement par des orpailleurs, de sommes d'argent oscillant entre 50000 et 100000 francs CFA constitue certainement la raison qui pousse ces jeunes à livrer leur corps aux orpailleurs dans de telles conditions.

\section{Conclusion}

L’exploitation artisanale de l'or est une activité largement répandue dans les localités d'Angovia, Allahou-Bazi et de Kouakougnanou. Cette activité dont l'origine remonterait au $18^{\mathrm{e}}$ siècle, selon les témoignages des anciens, a pris de l'ampleur à la fin du siècle dernier pour devenir aujourd'hui la principale activité socioéconomique des populations.

L'une des caractéristiques de l'exploitation artisanale de l'or au niveau du Yaourè est qu'elle est illicite, c'est-à-dire qu'elle n'a pas l'aval de la loi; en dépit de cette situation, cette activité mobilise de nombreux acteurs dont les plus nombreux sont des allogènes, principalement des burkinabés. On retrouve également dans la filière de l'orpaillage des personnes relevant de l'administration publique nationale. L'implication d'agents et cadres des services publics dans cette activité contribue à la perpétuer et lui donner une certaine légalité aux yeux des masses rurales.

Les impacts positifs de l'exploitation artisanale clandestine de l'or dans le Yaourè sont d'ordre économique; en effet, comme le disait un notable à Angovia, "chacun gagne pour lui" pour confirmer que chacun trouve son compte (économiquement parlant) dans cette activité, sauf, bien entendu l'Etat de Côte d'Ivoire; en effet, celui-ci ne perçoit aucune taxe de ce secteur qui semble générer d'importants revenus financier.

Les effets négatifs de l'exploitation artisanale clandestine de l'or sont très nombreux et concernent tous les domaines de l'environnement (environnements physique, biophysique et humain). Au plan humain, outre les problèmes classiques inhérents à une croissance rapide et incontrôlée de la population, l'exploitation artisanale clandestine de l'or sape les bases de l'économie rurale, fragilise les équilibres sociaux et communautaires et renforce la précarité des groupes vulnérables.

$\mathrm{Au}$ regard de ses différents impacts sur les composantes de l'environnement, l'exploitation artisanale clandestine de l'or apparait comme une activité qui ne peut induire un développement durable dans les localités où elle a lieu; aussi, l'Etat de Côte d'Ivoire, à travers les dispositions de la loi 
$n^{\circ}$ 2014-138 du 28 mars 2014 portant code minier, a-t-il soumis l'exercice de cette activité à des conditions de nature à en réduire les effets néfastes sur l'environnement.

Le développement de l'exploitation artisanale de l'or à caractère illicite apparait comme la conséquence de la conjugaison d'au moins trois facteurs sociaux: l'implication (directe ou indirecte) dans l'orpaillage des personnes censées faire respecter la loi, la mévente des produits agricoles traditionnels (cacao et café) et l'ignorance des impacts négatifs à moyen et long termes de l'orpaillage par la plupart des acteurs impliqués dans la filière. La lutte contre l'orpaillage clandestin ou "encadrer l'exploitation artisanale de l'or ${ }^{11 "}$ ne peut avoir les résultats escomptés que si l'on agit de manière durable sur ces facteurs.

\section{References:}

Actualité (2014, 5 mars). Côte d'Ivoire: le gouvernement s'engage à organiser l'orpaillage. Repéré à http://intellivoire.net/cote-divoire-legouvernement-sengage-a-organiser-lorpaillage.

2DConsulting (2015): Etude d'Impact Environnemental et Social (EIES) du projet de mine d'or du Yaourè, Amara-Mining, Abidjan.

CECAF International (2007): Etude d'Impact Environnemental et Social (EIES) du projet de mine d'or d'Angovia, Cluff Gold, Abidjan.

Jaques E. (2001): La mine artisanale en Afrique : aspects techniques et environnementaux.

CIFEG Publication 2001/37, p. 87-93.

Joseph Gaston J. (1913): Exploitation indigène de l'or en Côte d'Ivoire. In: Bulletins et Mémoires de la Société d'anthropologie de Paris, $\mathrm{VI}^{\circ}$ Série. Tome 4 fascicule 3-4, pp. 372-375.

Jacqueline Remits J. (2001) : Des mines et des hommes au pays de Liège, Edition du Céfal.

Joël Michel J. (1993) : La mine dévoreuse d'hommes. Paris, Edition Gallimard.

Kouadio K. N. (2008) : Exploitation artisanale de l'or dans le processus de mutation socioéconomique à Hiré (sud Bandama, Côte d'Ivoire) Université de Bouaké -D.E.A Sociologie.

Lebrun F. et Zanghellini V. (1981) : Histoire et civilisations, classe de seconde, Belin, p. 228

Niangoran-Bouah G. (1978) : Idéologie de l'or chez les Akan de Côted'Ivoire et du Ghana. In: Journal des africanistes. tome 48 fascicule 1. L'or dans les sociétés Akan. pp. 127-140.

${ }^{11}$ C'est l'expression utilisée par les autorités ivoiriennes pour parler de la lutte contre l'exploitation artisanale clandestine de l'or. 
Organisation internationale du travail (OIT) (1999) : Social and Labour Issues in Small-scale

Mines. Rapport soumis aux fins de discussions à la Réunion tripartite sur les problèmes sociaux et de travail dans les petites exploitations minières, BIT, Genève.

Patrice C. et Hoffmann G. (2000): Au temps des premières usines, Bruxelles, Edition Casterman.

Philippe Godar P. (2001): La vie des enfants travailleurs pendant la révolution industrielle. Paris, Edition du Sorbier.

Seydou Keita (2001) Etude sur les mines artisanales et les exploitations minières à petite échelle au Mali. 